\title{
A Paradoxical Way of Communication in Children With Autism Spectrum Disorders Syndrome
}

\section{Bujar Hoxha ${ }^{a}$}

\begin{abstract}
This paper seeks to exemplify some of the intentional and unintentional nonverbal communication attempts expressed by children with Autism Spectrum Disorder (ASD) syndrome. It demonstrates that such interactions with the rest of the world are trying to establish some semiotic functions, in an effort behaviorally to overcome the children's disability. The author's aim is to analyze such a paradoxical way of communicational interaction of such a subject vs. a given objectively perceivable reality, thus attempting to reveal paradoxical social contexts. The focus is on interpersonal communication abilities and/or disabilities, thus allowing semiotically distinguishable units as an object of analysis. The problem of mental disorders, such as ASD, requires a multi-dimensional approach-e.g., biological, medical, and other related fields. In conclusion, by applying a semiotic analysis, it becomes necessary to communicate with such children in a whole new way which, by processes of transformation, can bring about a state of awareness of a paradoxical and/or abnormal phenomenon.
\end{abstract}

\section{Keywords}

Autism, semiotics, transformation, passions, deficits

The term "communication", of a Latin origin, is primarily understood as an informational exchange and/or processing. It is not applied in terms of human behavior and its stratification into various social layers only, but it is also used as a tool of interconnecting media, to try and assist a permanent and uninterruptable process of communication within its distinguishable units. If such a view is taken to be true, then one should be aware of the term's complexity, explainable initially by the various analytical fields of communication, which lead toward establishing various communication theories. In such circumstances, scholarly approaches become multifold and can be viewed as various treatable methodologies. One can treat them synthetically (owing to their exactitude) in the sense shown by scholars (Shannon and Weaver), whereby a relationship between two mainly opposed analytical items is established; socially, if one wishes to see the relatedness of each side individually and collectively (Bruke and Stets 2009); or psychologically, if one wishes to mediate a determined "physiologically intended context" so as to contribute to the relationships' formation (Piaget 1969; Schwartz, Luyckx, and Vignoles 2011). Finally, it is possible to treat them even semiotically, if one

aSouth East European University, Republic of Macedonia

\section{Correspondent Author:}

Bujar Hoxha, Department of Communication Sciences, the South East European University, Ul. Haska 12, 1000, Skopje, Macedonia

E-mail: b.hoxha@seeu.edu.mk 
wishes to comprehend various circumstantial conditions introduced by other contexts which initially may seem implicit or undisclosed, in a sense of "absent structures" (Eco 1968). Of course, these are not the only disciplines in which one can envisage such a term as communication, either foreseen by a theoretical paradigm, or by their practical applicability. In this contribution, the author will try to elaborate the term in its various shapes, for the sake of such a process's accomplishment in determined social contexts, which may specifically be seen as paradoxical communication abilities.

Theories (Griffin 2003) foresee an idealistic accomplishment of the process explained by the existence of its components which make such processes' applicability come true. It would therefore be logical to ask: How can such tasks be performed within human emotionality? Can we thus overcome exactness? Does it consequently mean that unrepairable sequences (such as obstacles, "noise”, lack of defense mechanisms, etc.) of the communication processes should be left unequivocal instead of their presumed equivocalness? This question would not apply only to inter- and intra-human behavior and/or its explicit manifestation status, but as well to all other types of the communicational processes performed: either belonging to the objectively found realities, or to a subjective shaping of such realities (which can, among other related issues, be exemplified within artistic expressions, as for instance, one of the possibilities). In such a fashion, one should identify such theoretical paradigms, which by hypothesizing their practical applicability can become an object of a determined multi-disciplinary approach. The aim in this paper is to examine some of the intentional and unintentional non-verbal communication attempts expressed by children with Autism Spectrum Disorder (ASD) syndrome, as well as showing their interaction with the rest of the world, thus trying to establish some of the semiotic functions (Piaget 1969) supposedly ready to overcome such children's disability. The author aims to analyze such a paradoxical way of communicational interaction of such a subject vs. a given objectively perceivable reality, thus attempting to reveal paradoxical social contexts. The focus is on interpersonal communication abilities and/or disabilities, thus allowing semiotically distinguishable units as an object of analysis. It should be observed that the problem (of such mental disorders as ASD syndrome) itself requires a multi-dimensional approach, e.g., biological and medical, among other related fields (Siegel 2003; Mash and Wolfe 2004). By applying a semiotic analysis, it becomes necessary to communicate with such children in a whole new perceivable way, which by the process of transformation can bring about a state of awareness of a paradoxical and/or abnormal phenomenon. Here, techniques and methodologies will be noted which may possibly modify such children's behavioral impairments.

Scientifically speaking, such processes identifying in terms of their applicability into an analytical elaboration of given hypotheses, require a simultaneous approach within some of the related disciplines. In this context, one should talk of the nonverbal kind of communication (including the disciplines involved), so as to be able to set given determined relationships among subjects ready to create a semiotically analyzable theoretical framework.

\section{VERBAL VS. NONVERBAL}

Each one of us maintains or possesses the ability to express himself/herself, at least, at the level of attempting, or wishing to do so. Some of us can perform such processes automatically; some others instead may need assistance to do so. In addition, one has to be aware of the fact that not everything can be expressed verbally, or vice versa, non-verbally. One can easily recognize the multi-dimensionality of the communication concept as such. 
One should consider that it covers conventionally established cultural categories such as is language [as, for instance, in linguistically-minded semiotics, but not only this (Samovar and Porter 2004)]. Second, it covers human interaction, as a tool directed toward creating a social reality and/or context ${ }^{1}$. Third, it covers human behavior, as an interaction with the self and others, as one of the traits related to our social responsiveness, as well as to our inter-human relationships. Finally, all such mentioned fields, may have their relational counter-parts, thus ready to create relations as one of the semiotic functions. If one considers such facts scientifically justifiable, communication (including social behavior as well), can be seen in terms of its various analytical levels. One has consequently to advance the following questions: In how many analytical levels can communication be analyzed? To what an extent is it inclusive or exclusive with a semiotic process? And finally, what do such processes have in common with the human impossibility of a verbal expression and/or with an unusual kind of behavior, in the sense that can be exemplified within the paradoxical social context of the individuals living with autism?

The juxtaposing of the two mentioned levels of communication is of a semiotic nature, because: First, gestures, mimics, eye-contact, visualization, cognition, etc. (human interaction, either performed consciously or unconsciously), above all represent an informational exchange, which can be seen either in the form of signs (thus, containing form and content, which may result in a determined meaningful unit), or can be already encoded signs (in the sense of metaphoric meaning: either socially acceptable or not), thus contributing to a process of signification. Second, such kinds of signification can render a multifold semantic spectrum, thus making distinguishable its basic units. Both processes represent core semiotic processes.

Such kinds of communication should be complementary to one another, so as to create a fully accomplished communicational process in distinction to what one may call meta-communication. In such cases when one cannot see such complementariness, one can envisage the impossibility of a verbal expression, as well as the process of instant or gradual de-contextualization and de-socialization. Such a juxtaposition of terms should be analyzed within their mutual exclusiveness or inclusiveness. Treating various communicational components (necessary for exemplifying such mental disorders as ASD is) out of a developmental viewpoint, shall show persuasively the gradualness of the procedures involved: Either in terms of inheriting habits and/or behavioral patterns (as a biological component), or, in terms of acquiring basic as well as complex psychological processes, thus enabling us to comprehend such states of an impossibility of relatedness and expressivity. One should define nonverbal communication as such, taken from its informational processing viewpoint, so as to be able to comprehend the stages of such habits and behavioral patterns evidenced by humans. Experiencing such a context demonstrates the indispensable communicative aspect of developmental psychology. Such a scientific concept is defined by some determined scientific fields. We shall therefore attempt to provide for some of them here. Prior to our defining certain aspects as theoretically explicable, let us see some of the behavioral impairments which are seen as a part of a "child's own world" in terms of labelling him/her within the spectrum.

\section{INTERPERSONAL COMMUNICATION AND ASD SYNDROME PHENOMENON JUXTAPOSED}

Interpersonal communication is said to transmit messages either verbally or non-verbally. Such kinds of communication should, theoretically speaking, include all other kinds of communicating possibilities that can be, and are, performed and/or lived by 
humans. Having proposed such a methodology in terms of comprehending the interpersonal communication concept, one has to be aware not only of the social aspects within, but as well of the psychological ones, which otherwise contribute to a rightful conceptualization of our existence.

The initial steps of human development (either taken from the biological or psychological point of view) do not include the verbal component. Such kinds of communication emerge as a necessity of our basic organic and biological functions. A baby's first cry when facing external reality is evidence of this. This has to do with processes of adapting oneself to the brand new surroundings, as well as to the process of contextualization with the newly faced social reality $^{2}$. Later on, other kinds of communication gradually appear, which certainly belong to our developmental patterns of behavior (Piaget 1969). There may come a later period when one can think in pictures [such as foreseen in individuals with ASD (Grandin 2008)], attempting thus to create overall perception and cognition processes ${ }^{3}$. We need not here attempt an analysis of the stages of human behavioral development in detail, but it is here appropriate to identify some of them for at least two reasons: first, to be able to comprehend what interpersonal communication is, especially from the psychological viewpoint; and second, to be able to determine what are the points (and/or the stages) when a child might delay acquiring such communicational and/or psychological processes, such as in children with mental disorders. In attempting to establish such a comparative view, one has to identify the behavior of a child suspected of having such impairments.

First, a child with autism shows atypical behavior instead of the typical in comparison to his/her biological age. Such behavior can be identified in the following way: performing actions of the same kind, such as stereotypically playing with one beloved toy in his/her own way. Such playing may become repetitive and unusual, and may last longer than usual.
Second, such a child does not maintain eye-contact upon call. He/she seems closed into his/her own "shell” or context. Third, such a child may not be capable of rightful perception and/or cognition of the objects around him/her, in the sense of determining their actual and or symbolic (reference) function. Fourth, such a child may not be social, i.e., may be escaping from various kinds of socializing with peers of his/her age. Fifth, such a child can have other neurological abilities, such as physical abilities in the sense of overcoming the biological age of his/her peers, by way of compensating for his/her mental disabilities. Of this last point, it would perhaps be more exact to suggest that such a child possesses an atypical neurological system (or such individuals possess neurodiversity). Such a state or situation is defined in the following way:

Autistic disorder or autism is a severe developmental disorder characterized by abnormalities in social functioning, language, and communication, and unusual behaviors and interests. It includes every aspect of the child's interaction with his or her world, involves many parts of the brain, and undermines the traits that make us human-our social responsiveness, ability to communicate, and feelings for other people (Mash and Wolfe 2004: 284).

In this situation, one has to conclude that the issue is multi-dimensional. Not only can communication be difficult, or at times even impossible, but it can also affect social behavior. Phrased differently, we may state that not only a counter-response that one may ask from such an individual is in question, but as well, his/her relatedness to others (or even to significant others) may be unclear ${ }^{4}$. Basing ourselves in such symptoms that the child and/or an individual might possess; we conclude that he/she still maintains the communication component. This may be a strange, unclear, tense one, full of anxiety, with possibilities of tantrums and/or meltdowns, and in terms of a possible common misunderstanding and/or misconception by the side of the significant others-yet it is still 
communication. We shall name such an example of communication within individuals (alongside, naturally, other socially interactive related issues) on the spectrum "a paradoxical one”. Such a paradoxical kind of communication analogously will create paradoxical social contexts, which may become passionate, and such passions may then by way of semiotic transformational procedures express feelings (such as: anger, disagreement, despair, happiness, joy, etc.) as final semantic units. The author will also attempt to single out such kinds of paradoxical communicational ability, which may come as a consequence of such individual's neurodiversity as opposed to a human neurotypicality and/or neurotypical individuals. One can see here the connection to such a phenomenon with the semiotic functions applied. After all, semiotics in the frames of its epistemological and discursive level, among other related issues - which as we said, see the phenomena as juxtaposed to one-another (Greimas and Fontanille 1993)—can foresee such explicable taxonomic units, when specifically speaking of a child having an autistic behavior.

Language, or better, verbal expressivity, as a final and a manifestation phenomenon (as only one of the many other noticeable deficits and/or paradoxes in the frames of the items discussed) is what such a child may partially or totally lack. As the author has observed elsewhere:

As we have stated above, instead of acquiring the basic knowledge of the world around, and the various objects in terms of their conceptualizing and differentiating in various categories and states, in short, of the social surroundings, a child with mental disorders can acquire only some of them. Advancing in some spheres, but not in all of them, is usually a characteristic of a child living with autism. (Hoxha 2014a: 219)

The problem here is compounding all such mental processes, or such different stages of perceiving objective reality, into one: or making them function toward a typical inter-personal communication. An atypical kind of communication is what occurs within such individuals, and is also due to sensory integration deficits and/or problems (Emmons and Andersen 2005). The author intends to emphasize those which may contribute to developing a semiotic function. In such respect, the construction of the social reality (in a close relation to our identity and/or identities formation) is what first should be elaborated here, for the purpose of having semiotic methods applied.

\section{CONSTRUCTING A SOCIAL REALITY}

The term "social reality" or "social context", refers to our own perceiving of the world around-that is, to what we see from the external world. The external world is not in the shape of our own shaping and/or imagining it to be. Similarly, external social reality differs from our own context or the way of our perceiving it (Bogdashina 2005). One may conclude this from the following: First, constructing a social reality, or seeing it as presented to us, is first of all a matter of our own psychology. We all perceive objects and subjects around us differently. There is no such a thing as an oneness in either perception or visualization processes. On the contrary: They all belong to otherness. Second, not only do we differently perceive, cognize, and then categorize reality in its various classes and subclasses, in terms of what one may call objective and/or objectified reality (Bourdieu 1982) basing ourselves on individual grounds both unconsciously and consciously, but we can perceive it the way we wish to see it. It therefore includes a twofold vision and/or perception: The one that is presented and/or it is literally perceived of, and the one that is interpreted. The first process should belong to psychology and the next to semiotics. After all, such matters are all based upon a gradual and individual experiencing of external reality, so that in later developmental stages, they become useable and functional. 
Are there any other ways of constructing it though? Do we see it only individually or does it include collectiveness? In this, not only personality formational processes (seen as individual cases of human psychological development) should be considered, but also social and/or socializing processes. This comprehensiveness naturally makes one's analysis of the phenomena described even more complex. It might be more precise to note that instead of experiencing the external reality individually, it becomes experienced collectively, due to social interaction and relatedness. Collective experiencing, after certain determined human developmental stages in early childhood, is essential for establishing conventional categories, applicable and lived by a normal social interactive context.

The next question to answer is: How should such a reality become paradoxical? Or: Where do we see its paradox? Is it reachable, and does it after all, overcome the conventionally established categories, as mentioned above? The answer to such questions leads one to the problem of those who live an exceptional context (Hoxha 2014a). Such a context, of course, is not seen only in individuals with ASD. The behavior indicated is visible, and is a subject to a different kind of perception of reality. In this, not only a special kind (or a paradoxical kind) of construction of reality can be noticed in other fields as well, but it is even lived and experienced — this is, above all, due to our overall interpretative capabilities. We use our senses to experience reality, so as to make it functioning, and finally, conventional. In conclusion, the aim is here to focus ourselves on such a paradoxical way of interaction within frames that can be exemplified within individuals labelled on the spectrum.

\section{Conceptualizing a Paradoxical Social Reality}

We all use our senses to render the form and the meaning of everything surrounding us. A process of an overall integration of all such senses occurs. Moreover, we use the word "sensational" in cases of facing an object or subject that has for a certain period and/or moment of time, occupied all our senses. Such a situation can be instanced, for example, within the witnessing of a work of art (which has a component of uniqueness that makes it different from the rest of external reality $)^{5}$. Yet how does an individual on the spectrum sense, categorize and conceptualize such a reality?

The answer to this question is complex. There are several reasons for this: The word "spectrum" implies the multiple phenomena that are visible and may be easily recognizable. Such phenomena, in addition, are not identical for all; or better, they do not belong all to oneness. Here, not only is an individual on the spectrum different because of possessing neurodiversity, but in addition each such individual, if compared with another, may behave differently in various circumstantial conditions. Such symptoms may be characteristic of a general kind of behavior in individuals with ASD. Or, as it is rightfully written in DMS V (Diagnostic and Statistic Manual of Mental Disorders), such a phenomenon possesses the following criteria:

(1) Persistent deficits in social communication and social interaction across multiple contexts, in areas described below, that the individual currently displays or did in the past:

(a) Deficits in social-emotional reciprocity (for example, failure to carry on a back-and-forth conversation, failure to imitate or respond to social interaction, nor having appropriate social approach behaviors);

(b) Deficits in nonverbal communicative behaviors used for social interaction (for example, abnormalities in eye-contact and body language; lack of facial expressions);

(c) Deficits in developing, maintaining, and understanding relationships (for example, difficulties in adjusting behavior to suit various contexts, difficulties in sharing imaginative play or making friends). 
(2) Restricted, repetitive patterns of behavior, interests, or activities as demonstrated by two of the following, that the individual currently displays or did in the past:

(a) Stereotyped or repetitive motor behaviors, use of objects, or speech (for example, echolalia);

(b) Insistence on sameness, inflexible adherence to routines, or ritualized patterns of verbal or nonverbal speech (for example, difficulties in moments of transitions, insistence on same food);

(c) Highly restricted, fixated interests that are abnormal in intensity or focus (for example, strong preoccupation with or attachment to unusual interests or objects);

(d) Hyper- or hyporeactivity to sensory input or unusual interest in sensory aspects of the environment (for example, does not appear to feel extreme cold or heat, excessive touching or smelling of objects) (Sicile-Kira 2014: 30-31).

As can be seen, the manifestation status of an individual possessing such behavior has been instanced. There may be many causes and hypotheses of such a kind of manifestation level, and/or of a conceptualizing such a social reality. An issue that has to be discussed here first, though, is that which is followed by and/or that represents a consequence of these kinds of atypical behavior (sensory integration deficits). These will seem unbalanced, and/or disproportional. For instance, instead of the actions of doing, such as performing various kinds of simple commands, the child may possess auditory or visual sensory needs. If all such sensory inputs, and/or stimulations from the external reality (and/or from the child's brain impulses) come at once, or, are contemporarily witnessed by a child (in the fashion, naturally that the child might perceive them), then such an individual might experience sensory overload. If such stimuli come from everywhere, or are too much for such an individual, then he/she may become anxious to be released from them: Again, by performing stereotypical or atypical actions, which are usually seen in the shape described in the citation above. In order to get a "communicative" answer to such interactional attitudes of an individual on the spectrum, thus to be able to have a clear-cut picture of his/her paradoxical social reality, one has to ask: What does the sensory integration phenomenon represent? Emmons and Andersen (2005) observe:

Sensory integration is a child's ability to feel, understand, and organize sensory information from his/her body and his/her environment. In essence, sensory integration sorts, orders, and eventually puts all individual sensory inputs together into a whole brain function. When the functions are whole and balanced, body movements are highly adaptive, learning is easy, and (good) behavior is a natural outcome. Sensory integration is also reflected in a child's development, learning and feelings about himself/herself. The connection between sensory integration and social and emotional development should not be underestimated. How a child integrates through the sensory systems provides a basis for his/her reality. Not your reality, not my reality, his reality - and his unique perspective on the world around him (Emmons and Andersen 2005: 14) ${ }^{6}$.

The uniting and/or compounding of all such information (which can be seen as stereotyped and repetitive activities in terms of individuals within the spectrum, or as any other form as described in the previous citation) is what such an individual may lack. An integration process in the communicative sense of the word means an equivocal information processing. From the communication and semiotic point of view, such a process which we name communicational, should contain equivocalness, uniqueness, and proportionality of the transmitted material, ready to be processed, throughout all its constituent components. This is the way to make such a process meaningful, as a result. Similarly, an individual on the spectrum possesses and/or creates his social reality and/or context which we named paradoxical, in a special way, i.e., by way of mediating his communication 
abilities/disabilities through sensory integration problems, due to an inadequate informational brain processing. In such a context, one should presume the necessity of mediation in order to fulfill an accomplished communicational and semiotic process.

\section{AN ATTEMPT TO ESTABLISH A SEMIOTIC FUNCTION IN INDIVIDUALS WITH ASD}

Owing to the multi-dimensional nature of the issue discussed-be it a neurological, psychological, and a semiotic one-it should be understandable that the application of the both-sided communicational process, and/or of a semiotic function, is a difficult task in the frame of overall communicational deficits within the ASD phenomenon. The processing of the information, which should be in a permanent flow on both sides of the communication channel, is either interrupted or is not processed in a rightful way. Thus, a process of mediation is necessary, so as to make a determined semiotic process, and/or a communicational attitude functional. Other treatable methodologies (such as previously suggested, such as biological and medical) shall not be discussed here $^{7}$.

In terms of psychological grounds, one has to introduce the therapist, who plays the role of a mediator in a communication process, including all parts involved. This person may be a parent, or any other subject or object with which the child may be attempting to acquire social reality. It is worthy of note, however, that not only should such a child be contextualized, but should also be ready to possess a semiotic function, in the sense of differentiating objects and subjects surrounding him/her ${ }^{8}$. In order to access such a process behaviorally, several techniques should be used. The author considers two of them here: PECS (Picture Exchange Communicative System) and ABA (Applied Behavior Analysis) (Sicile-Kira 2014; Bogdashina 2005). Both techniques and/or methodologies to approach an individual labelled on the spectrum rely on behavioral acquisition of the contextual circumstances, necessary for a rightful perceiving of external reality and/or are aimed to a normal and neuro-typical human functioning. Besides psychological reasons, the techniques are semiotically relevant as well. If successfully applied, one can then differentiate between intentional and unintentional communication attempts of such children, but the interrupted communication process can also be intermediated and regained. Allowing the child to guess the right objects and/or subjects, then, after visualizing them, to categorize them, as well as to express them verbally, accomplishes the semiotic dichotomy between the signifier and the signified (Saussure 2011). All such processes which, in other social contexts, can be intended as automatically performed and/or processed, in terms of individuals possessing neurodiversity should be seen as separate processes (or, perhaps better, such ones that should be performed one after another). Prior to explicating the applicability of a semiotic function in such a context (a relation, a signification and/or establishing a narrative structure); let us first attempt to explicate the mentioned acquiring techniques. Bogdashina (2005) notes:

The PECS was developed by Dr. Andrew Bondy, a psychologist, and Lori Frost, a speech and language therapist in the Delaware Autistic Program, to help individuals with autism and other developmental disabilities acquire communicative skills (Bondy and Frost 1994). This system is based on principles of intentional communication, worked out long ago before PECS was introduced. What PECS does is to introduce these principles as a package, to be used in a systematic and prescribed way. PECS has been proved very successful for those who either do not use (and/or understand) speech or are echolalic (Bogdashina 2005: 213).

Instead of a self-initiated intentional way of communication, a child on the spectrum may 
communicate unintentionally, or even unexpectedly. All kinds of nonverbal communication should be included here: Be they certain unconscious movements (based on neurological impulses), or conscious ones, based on a self-initiative (such as body language, eye-contact, internalized speech attempts and behavior, etc.). The system explained represents an attempt to make their communication intentional and meaningful, for the rest of the world. All such children's actions are meaningful for their own context, for their own created paradoxical social reality. The aim of the technique, as well as the intervention of the specialist (or the active/or knowable subject) $^{9}$, is to offer the child both-sided communication abilities and a complete contextualization process.

A process of gradual acquisition through pictures, in a structured way, is what occurs within the usage of such a technique - not only that the child intentionally chooses a rightful picture, but he/she can also be shown actions of doing, which in later stages, aim to become functional. Semiotically speaking, it should imply the following: Each seen and/or visualized object (shown in a picture) then becomes united with its meaning - the first presented chaotic sign becomes motivated. Besides its motivation, the child, instead of literally perceiving the object, gradually attempts to gain its cognitive and metaphoric importance. This last remark of course, may require that other techniques are used, such as $\mathrm{ABA}^{10}$ :

To teach language to autistic children, Lovaas (1966) employed a "behavior modification" procedure, based on reinforcement learning theory and shaping techniques to develop a "program for the establishment of speech in psychotic children" (Lovaas 1966). The methods of this program include discretetrial training, incidental teaching, the natural language paradigm, and time delay. The Lovaas approach (also known as Applied Behavior Analysis or $\mathrm{ABA}$ ) is very well-documented (both favorably and unfavorably) (Bogdashina 2005: 248).
The process of changing and/or transformation of behavior would mean reducing sensory deficits, instructing the child to get contextually accustomed with the therapist, and teaching relational attitudes, as three early steps toward gaining a full communicative status. All of these represent a starting point to initiate a process of signification.

By conditioning the child through the ABA technique, in an attempt to gain modifications in his/her behavior, the child acquires context. This is an initial first stage of signification. Thus, not only can eye-contact perhaps be established, but as well as a relationship with the objects that the child wishes to use in terms of his/her context. Second, in later stages of acquisition, such a process of conditioning becomes substitutable or replaceable by other actions, which should belong to a rightful conceptualization of the subjects and objects around him/her. Instead of intermediating his/her actions through sensory integration deficits (such as proprioceptive, and/or visual or auditory), the child is gradually prompted toward using a determined object functionally. This process of so-called transition ${ }^{11}$ in acquiring new types of behavioral attitudes shall enable him to establish a semiotic function, and or a symbolic reference toward comprehending external reality.

Here the word "transformation" or "transformational" is of a crucial importance, from both the psychological and the semiotic viewpoint. The author numbers some reasons for this importance in relation to such an issue: first, psychologically, because of the step-by-step acquisition of new behavior. The rewarding, reinforcing, and/or punishing process led by the therapist is, for instance, crucial for achieving results. If one offers an object to such an individual, it should be conditioned with performing an action, such as showing attempts of recognizing it, and/or naming it, on the part of the child. If the results are positive, the patient should be rewarded. Such a step-by-step achievement of a behavioral modification can be reached after difficult 
attempts which shall foresee an improvement of the given situation. Second, semiotically, it is probable that each and every accomplished step represents a next stage of signification. For instance, recognizing an object represents a step toward the symbolic and representational stage of the patterns of behavioral development of the child (Piaget 1969). It automatically establishes a relationship between the patient and the therapist. Moreover, the next stages of such kinds of behavioral acquisition can move the process toward gradual encoding and decoding procedures, which are otherwise foreseen as basic semiotic processes ${ }^{12}$. Such kinds of newly acquired behavioral attitudes shall mark a second step of signification. By this assertion, the author includes not only perceptive capabilities (in terms of recognizing objects or subjects), but as well as cognitive capabilities (in terms of being able to differentiate and/or to guess the representational status of determined exemplified contexts in comparison to reality). The task of the therapist, in conclusion, is to offer the child different kinds of contexts (either in terms of "social stories", etc., or in terms of enacting his/her internalized speech ability), so as to enable him/her to develop receptive as well as representative skills, which most certainly include a semiotic function among other related issues.

To sum up, the relationship formation, as one of the initial issues of learning by such a child, creates what we call a semiotic function, not only in the shape of its perception and recognition on the part of the child, but also in the shape of including meaning. The repetitiveness in the process of teaching as a matter of fact, enables the child to develop short-term memory (STM), as opposed to long-term memory (LTM), (Goldman 1986), thus enabling him/her toward semantic units recognition in the semiotic sense of the word. Each repetition is an attempt to acquire reality in its structured and conventional shape (behaviorally, taxonomically, and finally, even emotionally), thus uniting such processes as elaborated above into one.

\section{CONCLUSIONS}

Because some semiotic processes lie on epistemological grounds, which can explain a manifestation of a semiotic function, it is important to state that in individuals on the spectrum, one can exemplify a process of reaching a peak of tensitivity ${ }^{13}$ in the frame of the semiotics of passions (Greimas and Fontanille 1993). Due to the fact that all such procedures have to rely on establishing a narrative process (and/or a narrative structure), one can envisage such situations in terms of experiencing some of the repetitive actions performed and manifested by such children. Their proprioceptive deficits, which are repetitive, suggest that such a manifestation contains its narration component. Such an action performed by them is meaningful, on one hand, in terms of having a well-planned strategy that is purposeful from the child's prospective, and, on the other hand, meaningless for the rest of the world (because of the fact that they belong to their paradoxical experienced and repetitive context). In such a context, a semiotic dichotomy can be established, one which would belong to the child's "physiologically acquired and accustomed context”, and the next, which would belong to the rest of social reality. This conclusion would lead us to the known dichotomy between appearance and reality. In appearance, such a child seems to lack meaning in terms of his/her suitability to the context(s) that a determined environment condition might challenge him/her. On the other hand, or in reality, such a child exhibits various communicational attempts, as we said, through sensory integration deficits, which seem meaningful only to his/her "world”. The semiotics of passions' task is to generate a trajectory of meaning units (be they passions, which might have emerged due to determined anxious situations lived by the child, or be they determined requests or complaints, which might seem incomprehensible to the significant others). Such 
a trajectory on both axes, which possess a relation of disjunction, must rely on epistemological and speculative grounds.

Due to such a lack of meaning, specifically between the child and his/her significant others, tensitivity occurs. In such a context, a modality in action should be enacted, so that a communication process is regained. Here the word "regained" is appropriate because of the symptoms and/or the paradoxical reality of a child possessing neurodiversity is seen, or is recognizable, approximately after the age of 18 months (Sicile-Kira 2014). Instead of the transition from the sensory-motor stage to the next ones, such as the pre-representational and the symbolic representational one (Piaget 1969), the child simply delays or remains at the mentioned stages, or still continues to possess sensory integration deficits.

When a significant other and/or a parent faces such a situation, or becomes aware of his/her child possessing delayed developmental progress, he/she becomes desperate. The passion of and/or the taxonomic notion of despair is what becomes obvious and noticeable here. Due to such a situation, the relation between the parent and/or the significant other and the child is disjunction.

Using a modality in action and/or enacting an active subject here, means enabling a transformational process aimed at substituting, and/or at replacing, activities offered to the child (be they of a reinforcement learning type, or of any other nature provided by the techniques previously mentioned), which would foresee an accomplishable communication process. The author purposely emphasizes the word "process" here, because in the frame of the child's physiological context, it does not function properly: In the sense that some of the signs ready for transmission actually remain unprocessed. Due to these deficits, the active and/or knowable subject replaces the child's performed actions by other ones or, specifically, by such actions which may be understood by his/her context(s). The term "replacement" and/or "substitution" in this context of our discussion is crucial: First, replacing movements, actions, and activities by new ones is a process which is usually done by the active subject (be it a parent or a therapist). Second, semiotically speaking, such a process creates the transformability process, in the frames of the inter-communication process between the child and the others, thus contributing to the syntagmatic axis in the Greimasian sense of the word. The replaced activity of the child (as, for instance, instead of mouthing an object as either a tactile or degustation sensory necessity of the child) gradually becomes displaced either by a functional usage of such an object, or by an expressivity of the child's passions. In conclusion, such an axis of seeming, as rightfully provided by the semiotics of passions, shall represent such a child's state of affairs, which, due to determined semiotic processes and/or behavioral interventions, is being transformed into an axis of reality, which shall for us represent the state of feelings (Greimas and Fontanille 1993). If the impaired activities of the child are not rightfully replaced by functionally using an object and/or referring to a subject, then he/she shall be subdued to the passions noted. Taxonomically speaking, such passions as anger, joy, a wish, and/or a compliant, cannot be permanent, in the sense that one can identify them in other related or discussable contexts. Such children are still in the course of the physiological development of their brains. Consequently, such passions may be changeable throughout various developmental stages in early childhood. In conclusion, such a syntagmatic axis, which is competent for the substitution of taxonomic notions understood as semantic units and/or related passionate experiences, represents not only one of the semiotic functions applied, but also a following developmental stage within overall communicative abilities/disabilities within an individual labelled on the spectrum. It is for these reasons that the author 
considers that such sorts of manifestation performed by such children should represent developmental taxonomic and or semantic units.

\section{Notes}

1. Such theories, for instance can be exemplified by G. H. Mead's interactionist symbolism. See Griffin (2003).

2. This process is an on-going one, even at later developmental stages of a child, and/or in the frames of adults and/or already mature individuals. We always face new realities, and try to comprehend them and acquire them, and/or embed them to our own context. The situation within children with ASD in this respect shall be explained later in this paper.

3. Such processes shall be explicated later where defining the terms of the developmental stages and the semiotic functions needed for our overall analysis.

4. As far as this kind of inter-personal communication is concerned see: Schwartz, Luyckx, and Vignoles (2011).

5. The author has treated the semiotic status of arts elsewhere. See for instance: Hoxha (2014b).

6. Italics are quoted from the original.

7. The neurobiological nature of the problem shall not be discussed here, in part because of the fact that such matters are still being resolved. Behavioral change can, however, supposedly mark a change within an atypical kind of behavior, which would manifest finally a semiotic process accomplished.

8. See Piaget (1969) for details in relation to the semiotic function in normal behavioral contexts.

9. See Hoxha (2014a), Normality vs. Abnormlity in a Child's Behavior (A Semiotic Perspective).

10. We mention here some of the related psychological processes, owing to the fact, in many of the cases, a combination of these (and other similar) techniques of acquisition are usually used by specialists.

11. See Bogdashina (2005).

12. For such processes, see for instance: Eco (1979), Theory of Semiotics.

13. Here the author is referring to Greimas and Fontanille (1993).

\section{References}

Bogdashina, O. 2005. Communication Issues in Autism and Asperger Syndrome: Do We Speak the Same Language? London: Jessica Kingsley.

Bondy, L. and L. Frost. 1994. "The Picture Exchange
Communicative System.” Behavior Modification 25:725-744.

Bourdieu, P. 1982. Language \& Symbolic Power. Cambridge: Polity Press.

Bruke, J. P. and E. J. Stets. 2009. Identity Theory. Oxford: Oxford University Press.

Eco, U. 1968. La Struttura Asente:la Ricerca Semiotica E Il Metodo Strutturale (The Absent Structure: The Semiotic Research and the Structural Method). Milano: Bompiani.

—. 1979. Theory of Semiotics. Edited by T. A. Sebeok. Bloomington: Indiana University Press.

Emmons, P. G. and L. M. Andersen. 2005. Understanding Sensory Dysfunction Learning, Development of Sensory Dysfunction in Autism Specrum Disorders, ADHD, Learning Disabilities and Bipolar Disorder. Philadelphia: Jesica Kingsley.

Goldman, I. A. 1986. Epistemology and Cognition. Cambridge: Harvard University Press.

Grandin, T. 2008. Thinking in Pictures: My Life With Autism. New York: Vintage.

Greimas, A. J. and J. Fontanille. 1993. The Seiotics of Passions: From State of Affairs to States of Feelings. Minneapolis and London: University of Minnesota Press.

Griffin, E. 2003. A First Look at Communication Theory. Bosrton: McGraw-Hill.

Hoxha, B. 2014a. "Normality vs. Abnormlity in a Child's Behavior (A Semiotic Perspective).” European Scientific Journal 10(14):212-226. Retrieved June 1, 2014 (http://eujournal.org/index.php/esj/article/view/3404/3168).

— 2014b. "On the Borderlines of Semiotics (Objectivity vs. Subjectivity).” European Scientific Journal 10(17):224-243. Retrieved July 1, 2014 (http://eujournal.org/index.php/ esj/article/view/3559/3399).

Lovaas, O. I. 1966. "A Program for the Establishment of Speech in Psychotic Children.” In Early Childhood Autism, edited by J. K. Wing. New York: Pergamon Press.

Mash, E. J. and D. A. Wolfe. 2004. Abnormal Child Psychology. Belmont, CA, Australia: Thomson/Wadsworth.

Piaget, J. 1969. The Psychology of the Child. New York: Basic Books.

Samovar, L. A. and R. E. Porter. 2004. Communication Between Cultures. 5th ed. Edited by A. Mitchell. Belmont, CA, Australia: Wadsworth/Thomson Learning.

Saussure, F. de. 2011. Course in General Linguistics. New York City: Columbia University Press.

Schwartz, S. J., K. Luyckx, and V. L. Vignoles. 2011. Handbook of Identity Theory and Research (Google eBook). New York: Springer.

Shannon, C. E. and W. Weaver. 1948. A Mathematical Theory of Communication. Urbana: University of Illinois Press. 
Sicile-Kira, C. 2014. Autism Spectrum Disorder (The Complete Guide to Understanding Autism). New York: Penguin Group.

Siegel, B. 2003. Helping Children With Autism Learn: Treatment Approaches for Parents and Professionals. New York: Oxford University Press.

\section{Bio}

Bujar Hoxha, Ph.D., associate professor, Department of Communication Sciences, South East European University; research fields: theoretical and applied semitics, semiotics of passions, and semiotics of disabilities. 